\title{
Exploration: an overarching focus for holistic development
}

\author{
DAVID STODDEN ${ }^{1}$ | KIMBERLEY D. LAKES 2 | JEAN CÔTÉ ${ }^{3}$ | EIVIND AADLAND ${ }^{4}$ | VALENTIN BENZING ${ }^{5}$ | ALI BRIAN ${ }^{1}$ | \\ CATHERINE E. DRAPER ${ }^{6}$ PANTELEIMON EKKEKAKIS ${ }^{7}$ | GUIDO FUMAGALLI8 | ARTO LAUKKANEN9 | MYRTO F. \\ MAVILIDI10 | EMILIANO MAZZOL $11^{11}$ | ROSS D. NEVILLE ${ }^{12}$ | DONNA NIEMISTÖ99 | JAMES RUDD 13 | ARJA SÄÄKSLAHTI9 | \\ MIRKO SCHMIDT ${ }^{5}$ | PHILLIP D. TOMPOROWSKI ${ }^{14}$ | PATRIZIA TORTELLA ${ }^{15}$ | SPYRIDOULA VAZOU7 | CATERINA \\ PESCE ${ }^{16}$
}

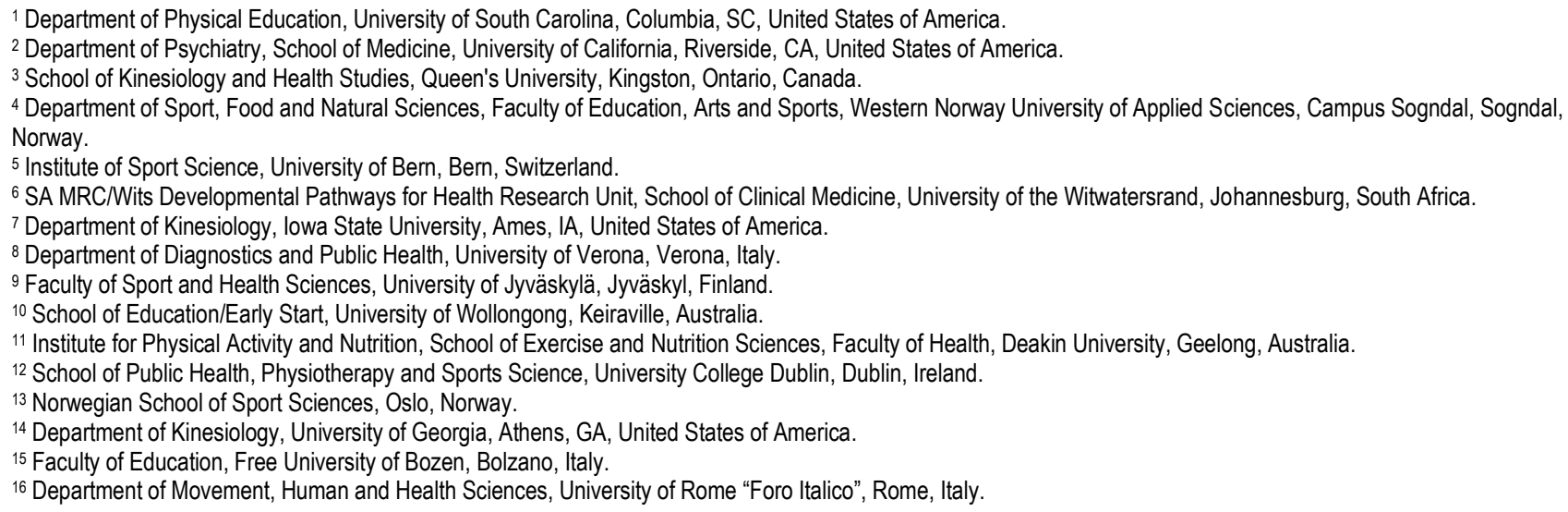

\section{ABBREVIATIONS \\ ADHD Attention-deficithyperactivity \\ WHO World Health Organization}

KEYWORDS: Children | Intervention | Holistic Development | Cognition | Play | Motor Development

\section{PUBLICATION DATA}

Received 27072021

Accepted 26082021

Published 01122021

\section{INTRODUCTION}

Promoting the development of health and well-being across the lifespan involves navigating a complex and intertwined labyrinth of biological, social, and environmental factors that change over time. Yet, approaches to positively impact developmental trajectories of these factors remain relatively siloed in academe and are generally disciplinespecific with a singular focus in their application (e.g., physical activity, motor development, social-emotional development, cognitive development). Discipline-specific interventions also are derived from a multitude of theoretical frameworks that are generally applied to only one domain of development. Attempting to amalgamate and apply various frameworks to promote holistic development would be useful, yet arduous and impractical based on our

\begin{tabular}{l|l|l|l|ll}
\hline Stodden et al. & 2021 & VOL.15 & N.5 & https://doi.org/10.20338/bjmb.v15i5.254
\end{tabular}


history of discipline-specific approaches. However, the process of identifying simplicity from complexity has been applied in many domains of science (e.g., neuroscience, biology, ecology, physics) in order to promote a more palatable, yet comprehensively encapsulating, conceptualization of complex mechanisms/phenomena. To quote Einstein, "It can scarcely be denied that the supreme goal of all theory is to make the irreducible basic elements as simple and as few as possible without having to surrender the adequate representation of a single datum of experience"1 (p. 165). Unfortunately, our siloed approaches to interventions designed to promote human development have, perhaps, been oversimplified and void of the essence of the complexity of human development. Quoting another eminent researcher (with a focus on human development), "It all comes back to the importance of action for learning and the fundamental interrelatedness of the different parts of the human being (the social, emotional, cognitive, and physical parts) and of all human beings to one another [...]. The best and most efficient way to foster any one of those (such as academic achievement) is to foster all of them"2 (p. 789). Thus, is it possible to provide a unifying theme for intervention that places an apriori focus on, and applies, an overarching catalyst of development to any intervention to promote holistic development?

The purpose of this commentary is to address this challenge, zooming out our view to encompass a variety of theoretical approaches without constraints on their different philosophical underpinnings. We propose a cross-boundary unification directive, converging on a critical component for any intervention that concomitantly illustrates the "why" and "how" of development that can be applied to any specific theoretical approach to this issue. Inherent in such a proposition would be the understanding that a unifying intervention directive should demonstrate a comprehensive understanding of human development, be applicable across any developmental domain, and be both justifiable and pragmatic. We propose that exploration should be this unified intervention directive for human intervention and provide evidence, and exemplars, that can be promoted from this simplistic unifying theme.

\section{The Far-Reaching Scope of Exploration}

Exploration is an innate motivation of all humans from birth and is our way of navigating and expanding our understanding of the complexity of the world. ${ }^{3}$ From a human development perspective, exploration involves the synergistic interaction of physical (i.e., motor), cognitive (i.e., learning to move and moving to learn), and other psychosocial (e.g., social-emotional) systems. As any volitional human movement can be fundamentally classified as "physical activity," our collective neuromotor (mind-body) system undergoes cumulative neurophysiological adaptations as we increase and refine our capability to explore our world. From infancy, the process of perceptuo-motor integration (via the use of multiple sensory systems) is a catalyst for continuing and advancing exploration of our environment and results in enhanced motor and cognitive skills.2,4 Thus, context-specific active exploration of our physical environment is spurred by the instinctive motivation to learn, ${ }^{5}$ via mastery experiences, ${ }^{6}$ and attain a goal. As young children age, new experiences continue to advance their development with the initiation of a new context for exploration socialization. In early childhood, competence in different movement forms (e.g., locomotor and object projection/manipulation skills), and cognitive and emotional skills (e.g., selfregulation, relying on both emotionally neutral - 'cool' - and emotionally laden - 'hot' cognitive executive functions) are fostered by and required to initiate social encounters and 
navigate social environments via structured and non-structured play. This development is enhanced in environments that allow for incremental challenges (i.e., the proximal zone of development described by Vygotsky). ${ }^{7}$

Unfortunately, research emphases on contemporary societal issues (e.g., sedentary behaviors, obesity, social-emotional deficits, academic achievement) have promoted siloed research that focuses on different facets of children's "health" rather than promoting holistic "development." Specifically, increased rates of obesity and associated metabolic diseases have prompted worldwide advocacy of an "exercise is medicine" research approach that has been propagated from adulthood down to childhood (i.e., a top-down age approach), which opposes a developmental approach (i.e., a bottom-up age approach). As a consequence, "play" is being replaced by medically based terminology such as "moderate-to-vigorous physical activity" and "exercise",8-11 thus removing the core values and the meaningful part of play from a child's perspective. In essence, a "quantity" of movement focusing on health outcomes, decontextualized from the indispensable features of play, has replaced the "quality" of children's movement and its associated holistic developmental outcomes. 10,12,13

\section{Exploration and Motor Development as a Foundation for Holistic Development}

While we appreciate the need to address the critical issues relating to the secular decline in children's (and adults') physical activity and health, this approach has overshadowed the critical need to focus on children's holistic development. For example, while research linking cardiorespiratory fitness and physical activity to children's cognitive health has received ample attention, knowledge of specific characteristics of interventions and the mechanisms underlying effects is limited. ${ }^{14}$ In essence, the causative mechanism for explaining brain growth (neurogenesis/synaptogenesis) and functionality (e.g., executive functions) has focused on metabolic pathways (e.g., angiogenesis); however, recent literature has suggested that cognitively engaging activities are more impactful on functional outcomes. ${ }^{15}$ Moreover, even when the "quality" of physical activity content and delivery is considered, the role of the context in which it occurs still remains largely neglected. Instead, it has been newly suggested that different causative mechanisms of physical activity's effects on the brain and cognition may be triggered - or not triggered - by physical, emotional, cognitive, and social features of the natural, virtual and built environments in which children participate in spontaneous or structured physical activities. ${ }^{16}$ Thus, our argument for understanding the context-specific nature of different types of physical activity (i.e., exploration) is critical to address how the brain develops and functions.

A mechanism that seems specific to the context of physical activity is cognitive engagement while moving. ${ }^{16}$ The execution of concomitant motor and cognitive tasks provides an added value to learning, outweighing potential acute negative effects of dual task interference. ${ }^{17}$ Motor and cognitive development are proposed to both be based on the same underlying mechanisms of skill acquisition; ${ }^{15}$ however, the beneficial effect of physical activity on learning may depend on the level of integration between the cognitive and motor tasks (i.e., temporal connection) and the relevance of movement complexity for the cognitive learning task (i.e., embodiment or relatedness). ${ }^{18}$ The additive effects of the combined motor and cognitive components are highlighted in embodied learning theories, advocating that cognition is situated in, and relies on, the interactions of the body with the external environment. ${ }^{19,20}$ In embodied learning, a child integrates information from different modalities (e.g., proprioceptive, visual, auditory), which are processed simultaneously by 
different cognitive systems, enriching memory traces during encoding and facilitating recall. ${ }^{21,22}$ More broadly, the emphasis of embodiment is put not on the body per se, but on the different bodily states, the perception of and interaction with the external environment, as well as the social, historical and cultural cues surrounding us. ${ }^{4}$

As children explore their physical and sociocultural environments, emotional development also unfolds as they engage in activities that involve both pleasure and discomfort, or pride and fear. ${ }^{23}$ Emotional development appears to be closely interconnected with cognitive development and this linkage is widely recognized where children are exposed to atypical caregiving experiences (e.g., parental separation, early institutionalization). Early negative emotional experiences may have profound negative effects on the development and integration of brain regions involved in cognition.24-26 The resultant dysregulation may have a lasting negative effect on the cognitive regulation of emotion, predisposing an individual to poor socialization and psychopathology. ${ }^{27}$ Thankfully, play and other vigorous activities elicit fun and enjoyment, positively impacting emotional and cognitive development, 28,29 including upregulation of neurotrophic factors and neurogenesis. ${ }^{30}$ However, the context again, must be called into play (pun intended). The context-specific nature of activities that children enjoy amplifies the importance of competency (e.g., motor competence) that is required for success and motivation for continued participation in developmentally appropriate activities.

The development of coordination and control (i.e., competence in movement) from infancy and its foundational role in physical development have been extensively documented (e.g., motor milestones). However, research noting its impact on positive developmental trajectories in other domains (e.g., physical health, cognition, self-concept, social-emotional development) across childhood and adolescence has received significant attention only recently. 31,32 Higher levels of movement skill are related not only to acute health-enhancing energy expenditure, ${ }^{33-35}$ but also to long-term physical activity, ${ }^{31,36}$ fitness, ${ }^{37,38}$ self-concept ${ }^{39}$ and weight status trajectories 37,40 across childhood and adolescence. Additional research is needed to understand potential differences in antecedent/consequent mechanisms that may change across time as psychological and cognitive processes involved in the development of coordination and control may have differing levels of influence based on age-related windows within the developmental continuum. ${ }^{41}$ In addition, positive trajectories of development in movement skills influence persistent engagement in preferred as well as novel activities (e.g., games and sports), and skills can be continually adapted to new environmental and social contexts. A critical antecedent to persistent engagement in activities is an individual's perception of competence ${ }^{39}$, which enhances motivation and confidence to successfully engage in activities with others. Thus, the impact that developing motor competence (or lack thereof) has on self-concept also influences social-emotional development. ${ }^{42}$ As Adolf and Hoch 4 note, "After all, motor development is really behavioral development [...]. Thus, for researchers who view psychology as the study of behavior, motor skill acquisition is the stuff of the science" (p. 157).

Data linking motor competence to multiple aspects of child development, $4,31,39,43,44$ as well as the burgeoning literature on "physical literacy" 45 and "long-term athletic development" 46 has also sparked a renewed focus on the necessity for a long-term holistic approach to child development research (i.e., a bottom-up approach). These emerging literature foci specifically address the importance of linking cognitive, self-concept, and social-emotional development via variability in learning experiences within different 
environmental contexts. ${ }^{32,47-51}$ In addition, promoting variability (i.e., exploration) in experiences with others speaks to children's engagement with and learning about individual differences (e.g., physical ability status, gender identity, race/ethnicity, and culture). Thus, variability in social experiences also may promote generational advances in the thought processes of developing citizens regarding equity, inclusion, and social justice. ${ }^{52}$

\section{Holistic Development for Every Child}

Considering what we have presented on the crucial facilitating role that exploration has on positive trajectories of physical, cognitive, psychological, and social-emotional development, it is imperative that every child be afforded opportunities to explore. While the World Health Organization (WHO) has recently published its first global physical activity guidelines for people with a disability, ${ }^{33,54}$ children with disabilities demonstrate increased odds of being excluded from engagement in structured physical activity opportunities. ${ }^{55}$ As a consequence, they are less likely to meet multiple health recommendations ${ }^{56,57}$ and various developmental milestones. For example, while school-/classroom-based strategies (e.g., active breaks, active lessons, standing desks) ${ }^{58}$ have been conducted to help children sit less and be more active, ${ }^{59-62}$ only a few studies have included children with disabilities. ${ }^{63,64}$ Although children with disabilities report enjoyment in various physical activities 65,66 and recognize them as opportunities for social interaction, ${ }^{67,68}$ they commonly perceive such occasions (even those delivered by experts) to be inappropriately designed and poorly matched to their abilities and needs. ${ }^{69}$ Compounding this issue in resource-poor settings, children with disabilities and/or developmental delays are generally not afforded the opportunity for referral and appropriate intervention. Many children 'fall through the cracks' of health and educational systems and stigmatization may result from a lack of insight and understanding of disabilities and how we need to increase inclusive practices.

Innovative and inclusive movement paradigms for all children are needed but are hindered by historical practices that are underpinned by medical models of disability and educational/social norms. Medical models focus on functional impairment (i.e., what children cannot do), thereby promoting an adaptive culture of sport and physical education that requires the child to "adapt" to the surroundings in order to be "included." In contrast, a social model of disability requires more flexibility in instructional methods and activities based on the needs, ability status and preferences of any learner in any context.70,71 For example, Universal Design for Learning makes adaptations readily available for all learners so that the learning experience is meaningful and relevant for each child. ${ }^{72}$ When children of varying needs and abilities engage collaboratively in inclusive movement experiences that promote embodied exploration in various contexts, ${ }^{73,74}$ all children can "learn" and thrive. ${ }^{75}$ Thus, identifying contextual factors that foster inclusion and equity, and their impact on socioemotional, cognitive, and motor domains is critical. ${ }^{76}$ Transitioning from exclusive into inclusive pedagogical practice will help overcome one of the main challenges to intervention - how to encourage teachers/practitioners/coaches to do more than simply set up a separate activity for the so-called 'disabled' child. Indeed, promoting inclusive strategies in any social, educational, and recreational setting and, at the same time, identifying and satisfying the requirement of individual differences is warranted, as they are not mutually exclusive, but complementary. For example, interventions tailored to address individual needs and competencies include emerging technology-based approaches, such as exergaming and integrative virtual reality. ${ }^{77}$ Specifically, exergaming research with children with attention- 
deficit/hyperactivity disorder (ADHD), autism, obesity, developmental coordination disorder and pediatric cancer survivors ${ }^{77-83}$ has demonstrated benefits within multiple domains of development..$^{77}$ As novelty, adaptivity, and variability are essential to elicit immersion and enjoyment, exergames in various virtual environments add to the array of pedagogical approaches to help foster exploration for all children.

\section{Exploration for Improving Intervention Science}

Identifying innovative pedagogical paradigms to promote holistic development necessitates several important shifts in how we may approach intervention and its implementation across a variety of contexts. The explorative approach should be extended from the child to the teacher/practitioner/coach level through a professional development process to develop interventions that meet their needs and contextual factors, as opposed to a prescribed standardized program. ${ }^{84}$ In addition, this paradigm requires that we explore novel measurement and analytical approaches to understand the impact of intervention on all individuals. Efficacy and effectiveness study designs are still dominated by ableism, as evidenced by the frequent exclusion of children with developmental and/or physical disabilities. This practice of "functional exclusion" 85 is in part a response to the demand for rigorous randomized controlled trials with strict inclusion and exclusion criteria and highly specified intervention protocols with little room for modification. Such rigorous approaches, rely on instruction mainly targeted to ensure 'what' and 'how much' is delivered (e.g., dose), whereas it is harder to control what children do when using more explorative interventions. Unfortunately, this approach might be mistaken for a weak intervention control, but it actually strengthens the capability to understand effectiveness of an intervention from an individual perspective. Further, as a consequence of word count limitations for many journals, too little information is provided on intervention protocols, fidelity of interventions, or the context in which the intervention takes place. Generally lacking in many intervention methodology sections is information on whether individuals participate equally across an intervention. While controlled trials may report on the number of sessions attended and may include data intended to be a proxy for participation (e.g., heart rate or accelerometry data during activities), the true quality of individual engagement (e.g., cognitive and socio-emotional engagement) is not captured and should be improved. 14,86 This problem can, perhaps, be a unique opportunity to globally enhance intervention research by not only improving intervention design, methodological protocols, intervention fidelity, and reporting of interventions, but also more effectively promoting inclusion. In essence, we need to promote interventions for all children and examine the impact of interventions using both "doseresponse" analyses that are based on person-centered approaches (e.g., regression based on individual engagement/practice trials/improvement) and traditional variable-centered analyses (e.g., intervention vs. control groups). This shift would enhance implementation science by promoting hybrid effectiveness-implementation designs. $87-89$

Moreover, adding a person-oriented analysis strategy ${ }^{90}$ to commonly applied variable-centered group analyses will not only help us understand what works for whom, but also will allow us to better map the complexity of holistic human development. ${ }^{91,92}$ This process enables researchers to disentangle true within-person intervention effects from group-level outcomes and moderators. ${ }^{93}$ Thus, rather than tightening inclusion criteria to reduce between-child variability, we could strive to be even more inclusive in our participant recruitment procedures and analyses by utilizing models that allow intervention intercepts 
(i.e., starting points) and slopes (i.e., changes over time) to be freely estimated and to vary at the individual as well as group level (e.g., using mixed fixed and random effects models). ${ }^{94}$ We may find that this approach identifies positive intervention effects where we may have otherwise thought there were no effects based on a collective group effect. ${ }^{95}$ In essence, does every child in the intervention group need to be a positive responder for the intervention to be deemed a success? Or, is it beneficial to identify the rate of effectiveness, particularly if this allows the intervention to be delivered in a more inclusive and equitable manner that provides all children with the opportunity to participate and explore the intervention environment? In our estimation, we believe the benefits of a "mandate for inclusion" in intervention research far outweighs the negatives as it represents a deeper level analytic approach, but more importantly, a more humanistic approach to science.

\section{Exploration at the Core of a Comprehensive Multilevel Approach}

For many children, early childhood education is the primary environmental context where they have opportunities to move, play, and learn beyond the home environment. Play and exploration are core concepts of early childhood education pedagogy, and their value for children's development (and learning) is undisputed. .,96-98 However, contemporary trends of "schoolification" and increased sedentary behaviors in young children are two worrisome trends that are counterproductive to children's development. 99,100 Facing these challenges, interventions with developmentally appropriate, child-centered (child-oriented, i.e., oriented to a child's needs) didactic approaches that integrate a focus on exploration via movement and play with the well-intentioned learning and health promotion objectives are wellpositioned to support broad educational goals and integrate public health initiatives during the early years.

Of course, to pursue such broader educational goals through active play, we need an educational alliance among key stakeholders who interact with children, such as parents, early care personnel, teachers and coaches; as well as a favorable social context within families and communities. ${ }^{101}$ Passionate adults communicate empathetically and emotionally with children using various levels of "scaffolding" actions to assist children in utilizing their imaginations and creativity during physical activities to develop problem solving and learning strategies. ${ }^{102,103}$ Exploring new environments often involves challenges that are necessary to promote continued development. As children engage in challenging tasks, adults are responsible for creating the physical and emotional conditions that help children find solutions, ${ }^{104}$ even if these represent "in itinere" achievements. Support and feedback from adults facilitate both motivation for continued engagement in challenging activities and perceptions of competence and enjoyment, and they also foster acceptance of momentary failures which plays an integral role in ultimately achieving the desired goal of learning and success. ${ }^{105,106}$ The integration of scaffolding processes in various contexts across a child's life, therefore, is critical to enhancing developmental processes in multiple domains. Outdoor environments also can provide a new challenge for children's development and can be viewed as a natural setting for scaffolding. ${ }^{107}$ Versatile outdoor environments offer plentiful stimuli for sensory systems and, at the same time, provide the possibility for children to explore how to use and control their body while searching for solutions on how to move. ${ }^{108,109}$ These outdoor environments may be planned/formal or natural, and adults can be catalysts who promote opportunities in outdoor environments (e.g., playgrounds, parks). These opportunities are critical to promote for all children, as children with disabilities and 
from disadvantaged environments can develop even in the absence of formal or intentional instruction or facilities. ${ }^{76}$ In addition, the child's perception of parental or other adult facilitated support for physical activity, specifically structure, autonomy support, and involvement, ${ }^{110}$ may facilitate internalization of autonomously regulated motivation for physical activity and enable confidence and competence for sustained participation and continued exploration of new activities and environments.

The school environment also provides opportunities for exploration in all areas of development. A collaborative effort to promote learning and health through the whole-school, whole-community, whole-child model ${ }^{111}$ highlights the need for schools to shift the focus from narrowly defined academic goals to goals that place holistic development of the child at the epicenter of education. Teachers, depending on the quality of their instructions, the challenges they provide, and the opportunities for interpersonal involvement they create, can positively or negatively impact motivation, enjoyment, and physical self-perceptions in students. In contrast, a non-supportive environment that thwarts the satisfaction of basic psychological needs may result in maladaptive motivational outcomes, such as disengagement, boredom, and reduced effort. ${ }^{14,112-114}$ As researchers assemble the missing pieces of exploration in school-based interventions, facilitators of and constraints to holistic development should be identified at several individual, interpersonal, and environmental levels.

Youth sport is another critical environment for holistic development, and a noteworthy framework that reinforces the importance of exploration is Côté and colleagues' Developmental Model of Sport Participation (DMSP). ${ }^{115,116}$ Within the DMSP, it is recommended that young athletes progress through stages of participation that include a period of sampling during childhood. The sampling years are characterized by a high level of diversity both within sports and between sports. Côte and colleagues ${ }^{117}$ proposed that the exploration of different sports and diverse activities within a sport help young athletes to: (a) increase their chances of acquiring a broad repertoire of skills and psychosocial assets as well as (b) decrease their likelihood of premature sport attrition resulting from injury and burnout. For example, children can play soccer on a beach with friends, in a park, or in an organized game with teammates and a coach. Although these three activities could be classified as playing soccer, the context-specific variability provides children with different ways to explore the sport in different physical and social environments. To better conceptualize these distinct sport experiences, Côté and Erickson ${ }^{118}$ suggested that practice and play activities in youth sport can be conceptualized along a continuum relating to how much instruction and input is vested by an adult (i.e., coach or parent) versus the child. At one end of the continuum are sport or lead-up activities where adults have minimal roles in providing instructions. On the other end of the continuum are sport activities in which adults set the direction and provide the instruction in a more structured environment. The shift from externally controlled to self-controlled activities is vital for exploration and the development of interest in a domain. ${ }^{119}$ Within the sport domain, young athletes are often placed in environments that are dictated by the agendas of adults. Such an overemphasis on adultcentered processes may undermine interest, creativity, and engagement. ${ }^{120}$ As such, adults should examine ways of encouraging youth-centered experiences such as play, instead of solely focusing on structured practice activities. Deliberate play has been used in the athlete development literature to describe child-centered activities that are inspired by formal sport games. ${ }^{116}$ Deliberate play differs from non-sport physical play activities of infancy or early 
childhood, ${ }^{121}$ specific pedagogical games or play designed by adults to improve performance (e.g., teaching games for understanding or play practice) $)^{122,123}$ and deliberate structured practice activities typical of organized sport. 124 It is play because the goal is enjoyment and deliberate because the activity is inspired by a structured sport activity. 118

Deliberate play activities at any level are characterized by two critical criteria that are both typical of any type of play activities and essential for exploration: (a) a high degree of novelty and unpredictability, and (b) flexibility in their structure and form. ${ }^{125}$ For example, children involved in deliberate play will often rearrange the composition of teams if one team is winning too easily. Younger, less skilled players are also often accommodated or allowed to play by different rules to minimize size and skill advantages, thus exhibiting the key characteristics of play activities. The focus of participation is on the act or process of competing (i.e., intrinsically motivated) while also keeping the game going in a fair and fun manner, rather than on the outcome of winning. As such, the participants themselves take ownership over the flexibility and negotiability of rules to maintain fun competition between players of differing abilities. By removing the structural barriers of organized sport and adapting rules to the specific contexts and situations, deliberate play then becomes the quintessential type of activity that promotes creativity and exploration in youth sport.

To advance and promote thought processes that place a primary emphasis on exploration across multiple intervention domains and environmental contexts (with and without adult participation), we must continue to effectively promote, propagate, and systematically progress (in a safe and appropriate manner) every child's journey in all aspects of their development. In recent years, ecological theory frameworks have been increasingly adapted in the movement sciences. At the heart of ecological theories, such as ecological dynamics, is an embodied and situated approach to the constant, reciprocal, and interactive relationship between the learner and what the environment (including other people) affords the learner. ${ }^{126}$ Across time, children become more sensitive to physical affordances (invariant features) and other children/adults to effectively interact within the physical as well as the social context of environments via a self-organizing process. ${ }^{127}$ As individuals become more intimately attuned to all aspects of a specific learning environment, a deeper level of practical knowledge, or 'know-how,' promoted by the action of "doing," progressively emerges. ${ }^{128}$ From an ecological perspective, research promoting holistic development necessitates a commitment to undertaking research that studies children's behavior as a complex and dynamic system. ${ }^{129}$ This systems approach can be understood as a continually evolving strategy where exploration is fundamental to promote a "best fit" to satisfy task requirements (i.e., movement goals) and is based on the interactions of the developing individual who functions within different physical and social environmental contexts. This continual process of functionally adapting one's action capabilities is the essence of development ${ }^{127,130}$ and is why children should always be encouraged to explore and interact with their surroundings.

\section{Forging a New Path}

Concluding with another metaphorical reference, U.S. President John F. Kennedy, when referring to the exploration of space, noted that "We choose to go to the moon in this decade and do the other things, not because they are easy, but because they are hard". ${ }^{131}$ Parlaying this language to child development, continued exploration of physical, cognitive, emotional and social aspects of an individual's ever-expanding world is a complex and many 
times a difficult process that requires substantial physical and mental effort, with success and failure inherently part of this developmental process. Thus, we contend that continually providing and promoting appropriately challenging experiences in diverse environmental and social contexts nurtures children's innate motivation and enjoyment associated with exploration and is an optimal solution that will encourage continual physical, cognitive, and emotional effort to learn and succeed (Figure 1).

\section{EXPLORATION}

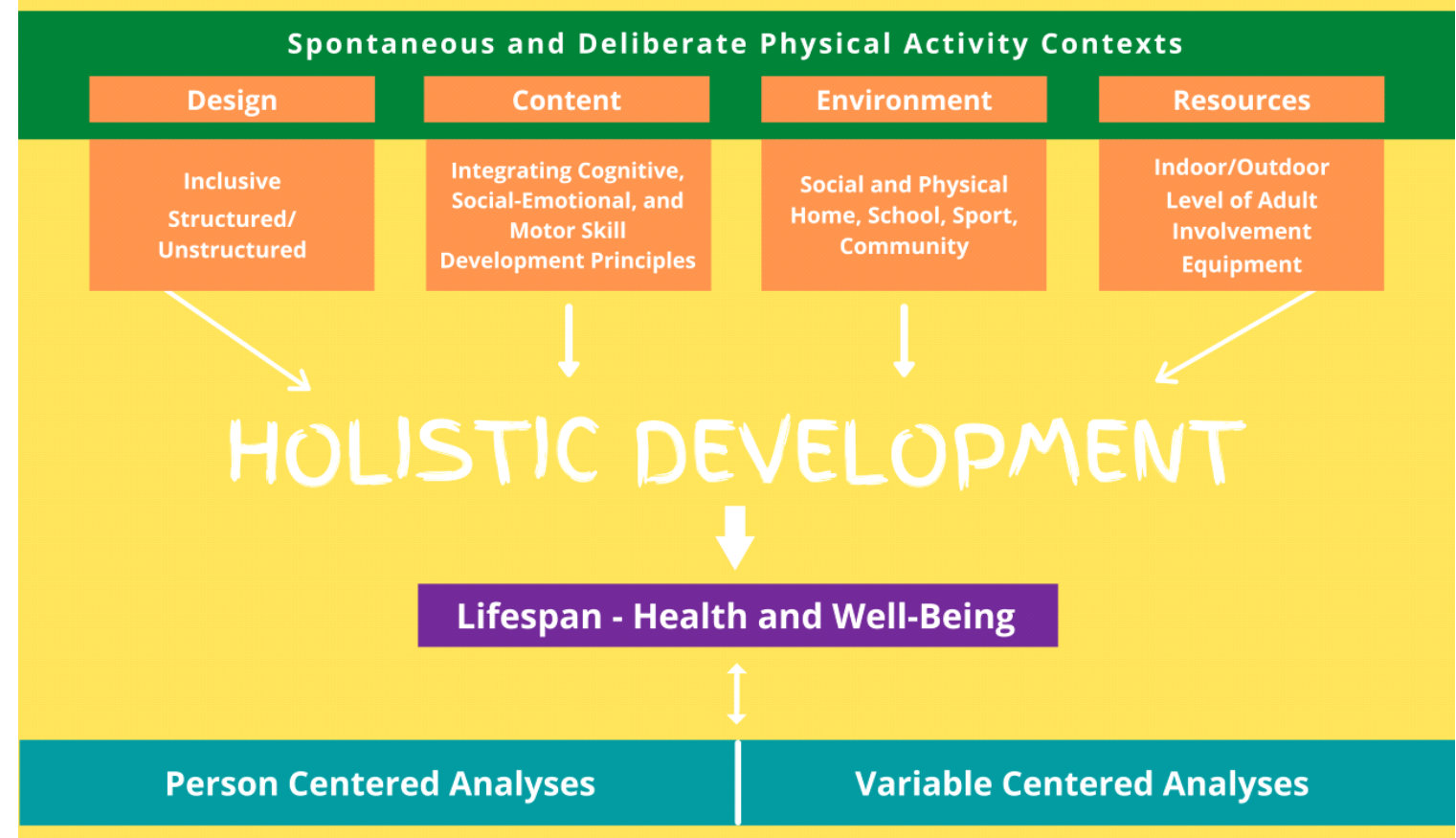

Figure 1. Flowchart of exploration context during child development.

We have started this cross-boundary paper on child development through physically active exploration stating that we wanted to overcome the limits of siloed thinking and fragmentary spirit that dominate discipline-specific research that does not address the complexity of whole-child development and of the ecological system in which it is embedded. In our proposed holistic perspective, however, we purposely avoided the notion of exploration as a conception of holism to intervention that may be seen as a 'reduction to the whole', thus to the same reductionism typical of individual disciplines intended to be opposed. Instead, we embrace a paradigm of complexity in its potentially simplest form. ${ }^{132}$ The overarching theme of promoting exploration as a principal component for any intervention, as has been described both metaphorically and with direct applications to all aspects of child development, provides a unifying phenomenological approach that bridges underlying foundations of current theoretical approaches in multiple domains to positively impact holistic development for all children. As the necessity to alleviate and potentially reverse continued negative trajectories of health and well-being that many children currently face, we hope we have effectively articulated a vision that provides the needed stimulus to re-think our approach to intervention by re-emphasizing a focus on holistic development. 


\section{REFERENCES}

1. Einstein A. On the method of theoretical physics. Philosophy of Science. 1934;1:163-169.

2. Diamond $A$. The evidence base for improving school outcomes by addressing the whole child and by addressing skills and attitudes, not just content. Early Education and Development. 2010;21:780-793.

3. Hedegaard M, Eriksen Ødegaard E. Children's Exploration and Cultural Formation. Springer Nature; 2020.

4. Adolph KE, Hoch JE. Motor development: Embodied, embedded, enculturated, and enabling. Annual Review of Psychology. 2019;70:141-164.

5. Schulz L. The origins of inquiry: Inductive inference and exploration in early childhood. Trends in Cognitive Sciences. 2012;16:382-389.

6. Bandura A. Self-efficacy: toward a unifying theory of behavioral change. Psychological Review. 1977;84:191.

7. Vygotsky LS. Imagination and creativity in childhood. Journal of Russian \& East European Psychology. 2004:42:7-97.

8. Alexander SA, Frohlich KL, Fusco C. Playing for health? Revisiting health promotion to examine the emerging public health position on children's play. Health Promotion International. 2014;29:155-164.

9. Alexander SA, Frohlich KL, Fusco C. Play, physical activity and public health: The reframing of children's leisure lives. Routledge; 2018.

10. Faigenbaum AD, Myer GD. Exercise deficit disorder in youth: play now or pay later. Current Sports Medicine Reports. 2012;11:196-200.

11. Milteer RM, Ginsburg KR, Mulligan DA. The importance of play in promoting healthy child development and maintaining strong parent-child bond: Focus on children in poverty. Pediatrics. 2012;129:e204-e213.

12. Pesce $C$, Faigenbaum AD, Goudas $M$, Tomporowski P. Coupling our plough of thoughtful moving to the star of children's right to play. Physical Activity and Educational Achievement Insights from Exercise Neuroscience. 2018.

13. Myer GD, Faigenbaum AD, Edwards NM, Clark JF, Best TM, Sallis RE. Sixty minutes of what? A developing brain perspective for activating children with an integrative exercise approach. British Journal of Sports Medicine. 2015;49:1510-1516.

14. Vazou S, Pesce C, Lakes K, Smiley-Oyen A. More than one road leads to Rome: a narrative review and meta-analysis of physical activity intervention effects on cognition in youth. International Journal of Sport and Exercise Psychology. 2019;17:153-178.

15. Tomporowski PD, Pesce C. Exercise, sports, and performance arts benefit cognition via a common process. Psychological Bulletin. 2019;145:929.

16. Pesce C, Vazou S, Benzing V, Álvarez-Bueno C, Anzeneder S, Mavilidi MF, et al. Effects of chronic physical activity on cognition across the lifespan: a systematic meta-review of 
randomized controlled trials and realist synthesis of contextualized mechanisms. International Review of Sport and Exercise Psychology. 2021:1-39.

17. Tomporowski PD, Qazi AS. Cognitive-motor dual task interference effects on declarative memory: A theory-based review. Frontiers in Psychology. 2020;11:1015.

18. Mavilidi MF, Ruiter M, Schmidt M, Okely AD, Loyens S, Chandler P, et al. A narrative review of school-based physical activity for enhancing cognition and learning: The importance of relevancy and integration. Frontiers in Psychology. 2018;9:2079.

19. Barsalou L. Situated simulation in the human conceptual system. Language and cognitive processes. 2003;18:513-562.

20. Mavilidi MF, Ouwehand, K., Schmidt, M., Pesce, C., Tomporowski, P. D., Okely, A. D., Paas, F. Embodiment as a pedagogical tool to enhance learning. In: Shapiro LS, S. (ed) In The Body, Embodiment, and Education: An Interdisciplinary Approach. Routledge; in press.

21. Risko EF, Gilbert SJ. Cognitive offloading. Trends in Cognitive Sciences. 2016;20:676688.

22. Wilson M. Six views of embodied cognition. Psychonomic Bulletin \& Review. 2002;9:625636.

23. Pollak SD, Camras LA, Cole PM. Progress in understanding the emergence of human emotion. Developmental Psychology. 2019;55:1801.

24. Gee DG. Sensitive periods of emotion regulation: influences of parental care on frontoamygdala circuitry and plasticity. 2016.

25. McEwen BS, Akil H. Revisiting the stress concept: implications for affective disorders. Journal of Neuroscience. 2020;40:12-21.

26. McEwen BS, Bowles NP, Gray JD, Hill MN, Hunter RG, Karatsoreos IN, et al. Mechanisms of stress in the brain. Nature Neuroscience. 2015;18:1353-1363.

27. Weissman DG, Bitran D, Miller AB, Schaefer JD, Sheridan MA, McLaughlin KA. Difficulties with emotion regulation as a transdiagnostic mechanism linking child maltreatment with the emergence of psychopathology. Development and Psychopathology. 2019;31:899-915.

28. Zhang B, Liu Y, Zhao M, Meng X, Deng Y, Zheng X, et al. Differential effects of acute physical activity on executive function in preschoolers with high and low habitual physical activity levels. Mental Health and Physical Activity. 2020;18:100326.

29. Rodriguez-Ayllon M, Cadenas-Sánchez C, Estévez-López F, Muñoz NE, Mora-Gonzalez J, Migueles $\mathrm{JH}$, et al. Role of physical activity and sedentary behavior in the mental health of preschoolers, children and adolescents: a systematic review and meta-analysis. Sports Medicine. 2019;49:1383-1410.

30. $\mathrm{Xu} \mathrm{J}-Y$, Chen $\mathrm{C}$. Endocannabinoids in synaptic plasticity and neuroprotection. The Neuroscientist. 2015;21:152-168.

31. Robinson LE, Stodden DF, Barnett LM, Lopes VP, Logan SW, Rodrigues LP, et al. Motor competence and its effect on positive developmental trajectories of health. Sports Medicine. 2015;45:1273-1284. 
32. Cairney J, Dudley D, Kwan M, Bulten R, Kriellaars D. Physical literacy, physical activity and health: Toward an evidence-informed conceptual model. Sports Medicine. 2019;49:371-383.

33. Sacko RS, Utesch T, Bardid F, Stodden DF. The impact of motor competence on energy expenditure during object control skill performance in children and young adults. Brazilian Journal of Motor Behavior. 2021.

34. Sacko RS, Nesbitt D, Mclver K, Brian A, Bardid F, Stodden DF. Children's metabolic expenditure during object projection skill performance: New insight for activity intensity relativity. Journal of Sports Sciences. 2019;37:1755-1761.

35. Duncan MJ, Dobell A, Noon M, Clark CC, Roscoe CM, Faghy MA, et al. Calibration and cross-validation of accelerometery for estimating movement skills in children aged 8-12 years. Sensors. 2020;20:2776.

36. Logan SW, Webster EK, Getchell N, Pfeiffer KA, Robinson LE. Relationship between fundamental motor skill competence and physical activity during childhood and adolescence: A systematic review. Kinesiology Review. 2015;4:416-426.

37. Cattuzzo MT, dos Santos Henrique R, Ré AHN, de Oliveira IS, Melo BM, de Sousa Moura $\mathrm{M}$, et al. Motor competence and health related physical fitness in youth: A systematic review. Journal of Science and Medicine in Sport. 2016;19:123-129.

38. Utesch T, Bardid F, Büsch D, Strauss B. The relationship between motor competence and physical fitness from early childhood to early adulthood: A meta-analysis. Sports Medicine. 2019;49:541-551.

39. De Meester A, Barnett LM, Brian A, Bowe SJ, Jimenez-Diaz J, Van Duyse F, et al. The relationship between actual and perceived motor competence in children, adolescents and young adults: a systematic review and meta-analysis. Sports Medicine. 2020:1-49.

40. Rodrigues LP, Stodden DF, Lopes VP. Developmental pathways of change in fitness and motor competence are related to overweight and obesity status at the end of primary school. Journal of Science and Medicine in Sport. 2016;19:87-92.

41. Stodden DF, Goodway JD, Langendorfer SJ, Roberton MA, Rudisill ME, Garcia C, et al. A developmental perspective on the role of motor skill competence in physical activity: An emergent relationship. Quest. 2008;60:290-306.

42. Pesce C, Stodden DF, Lakes KD. Physical Activity "Enrichment": A Joint Focus on motor competence, hot and cool executive functions. Frontiers in Psychology. 2021;12.

43. Haapala EA. Cardiorespiratory fitness and motor skills in relation to cognition and academic performance in children-a review. Journal of Human Kinetics. 2013;36:55.

44. Piek JP, Bradbury GS, Elsley SC, Tate L. Motor coordination and social-emotional behaviour in preschool-aged children. International Journal of Disability, Development and Education. 2008;55:143-151.

45. Edwards LC, Bryant AS, Keegan RJ, Morgan K, Jones AM. Definitions, foundations and associations of physical literacy: a systematic review. Sports Medicine. 2017;47:113-126. 
46. Pichardo AW, Oliver JL, Harrison CB, Maulder PS, Lloyd RS. Integrating models of longterm athletic development to maximize the physical development of youth. International Journal of Sports Science \& Coaching. 2018;13:1189-1199.

47. Orth D, Van der Kamp J, Memmert D, Savelsbergh GJ. Creative motor actions as emerging from movement variability. Frontiers in Psychology. 2017:8:1903.

48. Pesce C, Croce R, Ben-Soussan TD, Vazou S, McCullick B, Tomporowski PD, et al. Variability of practice as an interface between motor and cognitive development. International Journal of Sport and Exercise Psychology. 2019;17:133-152.

49. Rudd JR, Pesce C, Strafford BW, Davids K. Physical Literacy - A journey of individual enrichment: An ecological dynamics rationale for enhancing performance and physical activity in all. Frontiers in Psychology. 2020;11. doi: 10.3389/fpsyg.2020.01904.

50. Sylvester BD, Standage M, Dowd AJ, Martin LJ, Sweet SN, Beauchamp MR. Perceived variety, psychological needs satisfaction and exercise-related well-being. Psychology \& Health. 2014;29:1044-1061.

51. Sylvester BD, Standage M, Ark TK, Sweet SN, Crocker PR, Zumbo BD, et al. Is variety a spice of (an active) life?: perceived variety, exercise behavior, and the mediating role of autonomous motivation. Journal of Sport and Exercise Psychology. 2014;36:516-527.

52. Beelmann A, Heinemann KS. Preventing prejudice and improving intergroup attitudes: $A$ meta-analysis of child and adolescent training programs. Journal of Applied Developmental Psychology. 2014;35:10-24.

53. Bull FC, Al-Ansari SS, Biddle S, Borodulin K, Buman MP, Cardon G, et al. World Health Organization 2020 guidelines on physical activity and sedentary behaviour. British journal of Sports Medicine. 2020;54:1451-1462.

54. Di Pietro L, Al-Ansari SS, Biddle SJ, Borodulin K, Bull FC, Buman MP, et al. Advancing the global physical activity agenda: recommendations for future research by the $2020 \mathrm{WHO}$ physical activity and sedentary behavior guidelines development group. International Journal of Behavioral Nutrition and Physical Activity. 2020;17:1-11.

55. Rimmer JA, Rowland JL. Physical activity for youth with disabilities: a critical need in an underserved population. Developmental Neurorehabilitation. 2008;11:141-148.

56. Lai B, Lee E, Wagatsuma M, Frey G, Stanish H, Jung T, et al. Research trends and recommendations for physical activity interventions among children and youth with disabilities: a review of reviews. Adapted Physical Activity Quarterly. 2020;37:211-234.

57. Maïano C, Hue O, April J. Effects of motor skill interventions on fundamental movement skills in children and adolescents with intellectual disabilities: a systematic review. Journal of Intellectual Disability Research. 2019;63:1163-1179.

58. Salmon J, Mazzoli E, Lander N, Ayala AMC, Sherar L, Ridgers ND. Classroom-based Physical Activity Interventions. The Routledge Handbook of Youth Physical Activity. Routledge; 2020:pp.523-540. 
59. Bedard C, St John L, Bremer E, Graham JD, Cairney J. A systematic review and metaanalysis on the effects of physically active classrooms on educational and enjoyment outcomes in school age children. PloS One. 2019;14:e0218633.

60. de Greeff JW, Bosker RJ, Oosterlaan J, Visscher C, Hartman E. Effects of physical activity on executive functions, attention and academic performance in preadolescent children: a meta-analysis. Journal of Science and Medicine in Sport. 2018;21:501-507.

61. Mahar MT. Classroom-based physical activity and on-task behavior. Translational Journal of the American College of Sports Medicine. 2019;4:148-154.

62. Martin R, Murtagh EM. Effect of active lessons on physical activity, academic, and health outcomes: a systematic review. Research Quarterly for Exercise and Sport. 2017;88:149168.

63. Mazzoli E, Salmon J, Pesce C, Teo W-P, Rinehart N, May T, et al. Effects of classroombased active breaks on cognition, sitting and on-task behaviour in children with intellectual disability: a pilot study. Journal of Intellectual Disability Research. 2021;65:464-488.

64. Mazzoli E, Koorts H, Salmon J, Pesce C, May T, Teo W-P, et al. Feasibility of breaking up sitting time in mainstream and special schools with a cognitively challenging motor task. Journal of Sport and Health Science. 2019;8:137-148.

65. Leo J, Mourton N-E. According to the kids: Research from the perspective of children with disabilities. Routledge handbook of adapted physical education. Routledge; 2020:pp.432449.

66. Rekaa H, Hanisch H, Ytterhus B. Inclusion in physical education: Teacher attitudes and student experiences. A systematic review. International Journal of Disability, Development and Education. 2019;66:36-55.

67. Coates J, Vickerman P. Empowering children with special educational needs to speak up: Experiences of inclusive physical education. Disability and Rehabilitation. 2010;32:15171526.

68. Place K, Hodge SR. Social inclusion of students with physical disabilities in general physical education: A behavioral analysis. Adapted Physical Activity Quarterly. 2001;18:389-404.

69. Spencer-Cavaliere N, Watkinson EJ. Inclusion understood from the perspectives of children with disability. Adapted Physical Activity Quarterly. 2010;27:275-293.

70. Brian A, Miedema ST. Universal Design for Learning in physical education: Strategies for PETE. Teaching about social justice issues in physical education. 2019:85.

71. Makopoulou K, Thomas G. Educating teachers for effective inclusive pedagogies. Routledge handbook of physical education pedagogies. 2016:473-484.

72. Lieberman LJ, Grenier M, Brian A, Arndt K. Universal design for learning in physical education. Human Kinetics; 2020.

73. Holt L, Lea J, Bowlby S. Special units for young people on the autistic spectrum in mainstream schools: Sites of normalisation, abnormalisation, inclusion, and exclusion. Environment and Planning A. 2012;44:2191-2206. 
74. Stephens L, Ruddick S, McKeever P. Disability and Deleuze: An exploration of becoming and embodiment in children's everyday environments. Body \& Society. 2015;21:194-220.

75. Taunton SA, Brian A, True L. Universally designed motor skill intervention for children with and without disabilities. Journal of Developmental and Physical Disabilities. 2017;29:941954.

76. Howard SJ, Cook CJ, Everts L, Melhuish E, Scerif G, Norris S, et al. Challenging socioeconomic status: A cross-cultural comparison of early executive function. Developmental Science. 2020;23:e12854.

77. Benzing V, Schmidt M. Exergaming for children and adolescents: strengths, weaknesses, opportunities and threats. Journal of Clinical Medicine. 2018;7:422.

78. Benzing V, Spitzhüttl J, Siegwart V, Schmid J, Grotzer M, Heinks T, et al. Effects of cognitive training and exergaming in pediatric cancer survivors-A randomized clinical trial. Medicine and Science in Sports and Exercise. 2020;52:2293.

79. Benzing $V$, Schmidt $M$. The effect of exergaming on executive functions in children with ADHD: a randomized clinical trial. Scandinavian Journal of Medicine \& Science in Sports. 2019;29:1243-1253.

80. Fang Q, Aiken CA, Fang C, Pan Z. Effects of exergaming on physical and cognitive functions in individuals with autism spectrum disorder: a systematic review. Games for Health Journal. 2019;8:74-84.

81. Gao Z, Chen S, Pasco D, Pope Z. A meta-analysis of active video games on health outcomes among children and adolescents. Obesity Reviews. 2015;16:783-794.

82. Page ZE, Barrington S, Edwards J, Barnett LM. Do active video games benefit the motor skill development of non-typically developing children and adolescents: A systematic review. Journal of Science and Medicine in Sport. 2017;20:1087-1100.

83. Smits-Engelsman B, Vincon S, Blank R, Quadrado VH, Polatajko H, Wilson PH. Evaluating the evidence for motor-based interventions in developmental coordination disorder: A systematic review and meta-analysis. Research in Developmental Disabilities. 2018;74:72102.

84. Aadland E, Tjomsland HE, Johannessen K, Nilsen AKO, Resaland GK, Glosvik Ø, et al. Active Learning Norwegian Preschool (er) s (ACTNOW)-design of a cluster randomized controlled trial of staff professional development to promote physical activity, motor skills, and cognition in preschoolers. Frontiers in Psychology. 2020;11:1382.

85. Tripp A, Rizzo TL, Webbert L. Inclusion in physical education: Changing the culture. Journal of Physical Education, Recreation \& Dance. 2007;78:32-48.

86. Moore GF, Audrey S, Barker M, Bond L, Bonell C, Hardeman W, et al. Process evaluation of complex interventions: Medical research council guidance. BMJ. 2015;350.

87. Curran GM, Bauer M, Mittman B, Pyne JM, Stetler C. Effectiveness-implementation hybrid designs: combining elements of clinical effectiveness and implementation research to enhance public health impact. Medical Care. 2012;50:217. 
88. Cassar S, Salmon J, Timperio A, Naylor P-J, Van Nassau F, Ayala AMC, et al. Adoption, implementation and sustainability of school-based physical activity and sedentary behaviour interventions in real-world settings: a systematic review. International Journal of Behavioral Nutrition and Physical Activity. 2019;16:1-13.

89. Koorts H, Eakin E, Estabrooks P, Timperio A, Salmon J, Bauman A. Implementation and scale up of population physical activity interventions for clinical and community settings: the PRACTIS guide. International Journal of Behavioral Nutrition and Physical Activity. 2018;15:1-11.

90. Zibung M, Conzelmann A. The role of specialisation in the promotion of young football talents: A person-oriented study. European Journal of Sport Science. 2013;13:452-460.

91. Bergman LR, El-Khouri BM. A person-oriented approach: Methods for today and methods for tomorrow. New Directions for Child and Adolescent Development. 2003 Fall;(101):2538.

92. Yeh Y-C. Aptitude-Treatment Interaction. In: Seel NM (ed) Encyclopedia of the Sciences of Learning. Boston, MA: Springer US; 2012:pp.295-298.

93. Hamaker EL, Kuiper RM, Grasman RP. A critique of the cross-lagged panel model. Psychological Methods. 2015;20:102.

94. Hamaker EL, Muthén B. The fixed versus random effects debate and how it relates to centering in multilevel modeling. Psychological Methods. 2020;25:365.

95. Lakes KD, Neville R, Vazou S, Schuck SE, Stavropoulos K, Krishnan K, et al. Beyond broadway: analysis of qualitative characteristics of and individual responses to creatively able, a music and movement intervention for children with Autism. International Journal of Environmental Research and Public Health. 2019;16:1377.

96. Singer E. Play and playfulness, basic features of early childhood education. European Early Childhood Education Research Journal. 2013;21:172-184.

97. Ilgaz H, Hassinger-Das B, Hirsh-Pasek K, Golinkoff RM. Making the case for playful learning. International handbook of early childhood education. Springer; 2018:pp.12451263.

98. Ødegaard EE. Dialogical engagement and the co-creation of cultures of exploration. Children's Exploration and Cultural Formation. 2020:83.

99. Bingham S, Whitebread D. School readiness in Europe: Issues and evidence. International handbook of early childhood education. Springer; 2018:pp.363-391.

100. Driediger M, Vanderloo LM, Truelove S, Bruijns BA, Tucker P. Encouraging kids to hop, skip, and jump: Emphasizing the need for higher-intensity physical activity in childcare. Journal of Sport and Health Science. 2018;7:333.

101. Bronfenbrenner U. Child care in the Anglo-Saxon mode. Child care in context: Crosscultural perspectives. 1992:281-291.

102. Tortella P, Schembri R, Ceciliani A, Fumagalli GF. Dual role of scaffolding on motorcognitive development in early childhood education. Journal of Human Sport and Exercise. 2020;15(4proc):S1407-S1417. 
103. Fumagalli GF, Tortella P, Coppola R, Sgrò F. Physical or emotional scaffolding in a difficult motor task: What is better with 5-year-old children? Journal of Human Sport and Exercise. 2020;15(4proc):S1437-S1445.

104. Tortella P. The role of the educator/adult in supporting children of pre-school age in learning difficult tasks: the case of the Playground" Primo Sport 0246". Journal of Physical Education and Sport. 2019;19(S5):2015-2023.

105. Diamond A, Ling DS. Review of the evidence on, and fundamental questions about, efforts to improve executive functions, including working memory. Cognitive and working memory training: Perspectives from psychology, neuroscience, and human development. 2019:145389.

106. Ryan RM, Deci EL. Self-determination theory: Basic psychological needs in motivation, development, and wellness. Guilford Publications; 2017.

107. Niemistö D, Finni T, Haapala EA, Cantell M, Korhonen E, Sääkslahti A. Environmental correlates of motor competence in children-the skilled kids study. International Journal of Environmental Research and Public Health. 2019;16:1989.

108. Sääkslahti A, Niemistö D. Outdoor activities and motor development in 2-7-year-old boys and girls. Journal of Physical Education and Sport. 2021;21.

109. Sanderud JR, Gurholt KP, Moe VF. 'Winter children': An ethnographically inspired study of children being-and-becoming well-versed in snow and ice. Sport, Education and Society. 2020;25:960-971.

110. Laukkanen A, Aunola K, Korhonen E, Barnett LM, Sääkslahti A. Construct validity and reliability of the physical activity parenting questionnaire for children (PAP-C). International Journal of Behavioral Nutrition and Physical Activity. 2021;18:1-12.

111. Lewallen TC, Hunt H, Potts-Datema W, Zaza S, Giles W. The whole school, whole community, whole child model: A new approach for improving educational attainment and healthy development for students. Journal of School Health. 2015;85:729-739.

112. Curran T, Standage M. Psychological needs and the quality of student engagement in physical education: Teachers as key facilitators. Journal of Teaching in Physical Education. 2017;36:262-276.

113. Cuevas-Campos R, Fernández-Bustos JG, González-Cutre D, Hernández-Martínez A. Need satisfaction and need thwarting in physical education and intention to be physically active. Sustainability. 2020;12:7312.

114. Tessier D, Sarrazin P, Ntoumanis N. The effect of an intervention to improve newly qualified teachers' interpersonal style, student's motivation and psychological need satisfaction in sport-based physical education. Contemporary Educational Psychology. 2010;35:242-253.

115. Côté J, Vierimaa M. The developmental model of sport participation: 15 years after its first conceptualization. Science \& Sports. 2014;29:S63-S69.

116. Côté $J$. The influence of the family in the development of talent in sport. The Sport Psychologist. 1999;13:395-417. 
117. Côté J, Allan V, Turnnidge J, Erickson K. Early sport specialization and sampling. Handbook of Sport Psychology. 2020:578-594.

118. Côté J, Erickson K. Diversification and deliberate play during the sampling years.

Routledge handbook of sport expertise. Routledge; 2015:pp.305-316.

119. Hidi S, Renninger KA. The four-phase model of interest development. Educational Psychologist. 2006;41:111-127.

120. Larson RW. Toward a psychology of positive youth development. American Psychologist. 2000;55:170.

121. Pellegrini AD, Smith PK. Physical activity play: The nature and function of a neglected aspect of play. Child Development. 1998;69:577-598.

122. Griffin LL, Butler J. Teaching games for understanding: Theory, research, and practice. Human Kinetics; 2005.

123. Launder A. Play practice: the games based approach to teaching and coaching sports. Human Kinetics, Champaign, IL. 2001.

124. Ericsson KA, Krampe RT, Tesch-Römer C. The role of deliberate practice in the acquisition of expert performance. Psychological Review. 1993;100:363.

125. Lester S, Russell W. Play for a change: Play, policy and practice: A review of contemporary perspectives. National Children's Bureau London; 2008.

126. Gibson JJ, Gibson EJ. Perceptual learning: Differentiation or enrichment? Psychological review. 1955;62:32.

127. Rudd J, Woods C, Correia V, Seifert L, Davids K. An ecological dynamics conceptualisation of physical 'education': Where we have been and where we could go next. Physical Education and Sport Pedagogy. 2021;26:293-306.

128. Woods CT, Rudd J, Gray R, Davids K. Enskilment: an Ecological-Anthropological Worldview of Skill, Learning and Education in Sport. Sports Medicine-Open. 2021;7:1-9.

129. Thelen $E$. The ( $r e$ ) discovery of motor development: Learning new things from an old field. Developmental Psychology. 1989;25:946.

130. Davids K, Araújo D, Hristovski R, Passos P, Chow JY. Ecological dynamics and motor learning design in sport. Skill acquisition in sport: Research, theory and practice. 2012:112130.

131. Kennedy JF. We choose to go to the Moon. Speech presented at Address at Rice University on the Nation's Space Effort in Rice University, Houston (1962, September 12). 1963.

132. Morin E. From the concept of system to the paradigm of complexity. Journal of Social and Evolutionary Systems. 1992;15:371-385.

\section{ACKNOWLEDGMENTS}

We would like to thank Emma Lakes Kay for developing Figure 1. 
Citation Stodden D, Lakes KD, Côté J, Aadland E, Benzing V, Brian A, Draper CE, Ekkekakis P, Fumagalli G, Laukkanen A, Mavilidi MF, Mazzoli E, Neville RD, Niemistö D, Rudd J, Sääkslahti A, Schmidt M, Tomporowski PD, Tortella P, Vazou S, Pesce C. (2021). Functional Variability in Movement Coordination and Perception. Brazilian Journal of Motor Behavior, 15(5):301-320.

Editors: Dr Fabio Augusto Barbieri - São Paulo State University (UNESP), Bauru, SP, Brazil; Dr José Angelo Barela São Paulo State University (UNESP), Rio Claro, SP, Brazil; Dr Natalia Madalena Rinaldi - Federal University of Espírito Santo (UFES), Vitória, ES, Brazil.

Copyright:@ 2021 Stodden, Lakes, Côté, Aadland, Benzing, Brian, Draper, Ekkekakis, Fumagalli, Laukkanen, Mavilidi, Mazzoli, Neville, Niemistö, Rudd, Sääkslahti, Schmidt, Tomporowski, Tortella, Vazou and Pesce and BJMB. This is an open-access article distributed under the terms of the Creative Commons Attribution-Non Commercial-No Derivatives 4.0 International License which permits unrestricted use, distribution, and reproduction in any medium, provided the original author and source are credited.

Funding: This research did not receive any specific grant from funding agencies in the public, commercial, or not-forprofit sectors.

Competing interests: The authors have declared that no competing interests exist.

DOI: https://doi.org/10.20338/bjmb.v15i5.254 